\title{
Superheavy neutralinos as dark matter particles
}

\author{
Venya Berezinsky \\ INFN, Laboratori Nazionali del Gran Sasso, I-67010 Assergi (AQ), Italy \\ Institute for Nuclear Research of the RAS, Moscow, Russia \\ E-mail: Venya.Berezinsky@lngs.infn.it
}

\section{Michael Kachelrieß}

Institutt for fysikk, NTNU Trondheim, N-7491 Trondheim, Norway

E-mail: michael.kachelriess@ntnu.no

\section{Marius Aa. Solberg *}

Institutt for fysikk, NTNU Trondheim, N-7491 Trondheim, Norway

E-mail: marius.solbergentnu.no

\begin{abstract}
We propose the lightest supersymmetric particle (LSP) as a well-suited candidate for superheavy dark matter. The mass $m_{\chi}$ of the LSP should be above $3 \times 10^{11} \mathrm{GeV}$, so that gravitational interactions at the end of inflation can produce it with the correct abundance, $\Omega_{\chi} h^{2} \sim 0.1$. Weak interactions remain perturbative despite the large mass hierarchy, $m_{\chi} \gg m_{Z}$, because of the special decoupling properties of supersymmetry. As a result the model is predictive and we discuss elastic scattering of neutralinos on light fermions, self-scatterings and briefly annihilations within this scheme.
\end{abstract}

Identification of dark matter 2008

August 18-22, 2008

Stockholm, Sweden

\footnotetext{
${ }^{*}$ Speaker; based on the article Supersymmetric superheavy dark matter [1] by the same authors.
} 


\section{Introduction}

Uncovering the nature of dark matter (DM) is one of the most pressing problems of current research in particle physics and cosmology. A wealth of observational data suggests that a viable DM candidate has to be non-baryonic and should be non-relativistic, at least from the time of matter-radiation equilibrium on [2]. The various particles proposed as cold dark matter (CDM) candidates $X$ can be divided in two main sub-categories: Thermal relics were at least once during the history of the Universe in chemical equilibrium with the thermal plasma, while non-thermal relics have either sufficiently small interactions or a high enough mass $m_{X}$ to be never produced efficiently by processes like e.g. $e^{-} e^{+} \rightarrow X X$.

The present relic abundance $\Omega_{X}$ of a thermal relic scales approximately as $\Omega_{X} \propto 1 / \sigma_{\text {ann }}$ with its annihilation cross section $\sigma_{\mathrm{ann}}$. Moreover, unitarity of the $S$ matrix restricts annihilations into the $l$.th partial-wave of particles with relative velocity $v_{\text {rel }}$ as $\sigma_{\mathrm{ann}}^{(l)} \leq(2 l+1) 4 \pi /\left(v_{\mathrm{rel}} M_{X}^{2}\right)$ [3]. Since for non-relativistic point-particles higher partial-waves are suppressed, the observed value [4] $\Omega_{\mathrm{CDM}} h^{2}=0.105$ of the DM abundance constrains the mass of any thermal relic as $m \lesssim 100 \mathrm{TeV}$.

Two notable non-thermal DM candidates are axions and superheavy DM (SHDM). The original motivation to introduce in Refs. [5, 6] the latter possibility was to use the secondaries produced in SHDM decays as explanation for cosmic rays of the highest observed energies. Later it was realized that gravitational interactions at the end of inflation generate the correct order of magnitude for the DM abundance, $\Omega_{X} \sim 1$, for any stable particle with mass around $10^{13} \mathrm{GeV}$ and a reheating temperature $T_{R} \sim 10^{9} \mathrm{GeV}$ [7]. It is this generality of the production mechanism and its independence from the details of a concrete particle physics model that promotes SHDM to an attractive DM candidate.

Most of the previously suggested SHDM candidates belong to a new sector that has no treelevel interactions with standard model (SM) particles. By contrast, it is the aim of this work to study the possibility of having a SHDM particle with SM-like couplings to the weak gauge bosons. Since the longitudinal part of gauge bosons couples as $\propto g M_{X} / m_{Z}$ to a particle with mass $M_{X}$, weak interactions become generically strong for $M_{X} \gg m_{Z}$ and thus the perturbative expansion fails. Using partial-wave unitarity, Chanowitz, Furman and Hinchliffe [8] derived thereby an upper limit of $M_{X} \sim \mathrm{TeV}$ for particles coupling with SM strength to the weak gauge bosons. An exception to this bound are supersymmetric theories, if only mass terms are added that break supersymmetry (SUSY) softly [9], and in particular the minimal supersymmetric extension of the SM (MSSM) [10]. Therefore we are led to suggest superheavy supersymmetry, i.e. the case where all masses of supersymmetric particles are of order $10^{11} \mathrm{GeV}$ or larger, as a concrete model for SHDM with SM weak interactions.

Neutralino as LSP We assume throughout that the lightest neutralino $\chi \equiv \chi^{0} \equiv \chi_{1}$ is the lightest of the supersymmetric particles. The neutralino mass-matrix $M_{\chi}$ in the $\left(\tilde{B}, \tilde{W}^{0}, \tilde{H}_{1}^{0}, \tilde{H}_{2}^{0}\right)$ basis is given by

$$
\left(\begin{array}{cccc}
M_{1} & 0 & -c_{\beta} m_{Z} s_{W} & m_{Z} s_{W} s_{\beta} \\
0 & M_{2} & c_{W} c_{\beta} m_{Z} & -c_{W} m_{Z} s_{\beta} \\
-c_{\beta} m_{Z} s_{W} & c_{W} c_{\beta} m_{Z} & 0 & -\mu \\
m_{Z} s_{W} s_{\beta} & -c_{W} m_{Z} s_{\beta} & -\mu & 0
\end{array}\right)
$$


with $s_{\beta}=\sin \beta, c_{\beta}=\cos \beta$ where $\tan \beta=v_{1} / v_{2}$ is the ratio of the two Higgs VEVs, $s_{W}=\sin \vartheta_{W}$, $c_{W}=\cos \vartheta_{W}$ with $\vartheta_{W}$ as Weinberg angle, and $\mu$ as the Higgs mixing parameter.

The consequences of the limit $M_{1}, M_{2},|\mu| \gg m_{Z}$ for the neutralino has been already extensively discussed for a neutralino as thermal relic [11]. Neglecting the terms of order $m_{Z}$, the four neutralino mass eigenstates become a pure bino, wino, and the symmetric and anti-symmetric combination of the two higgsinos, $\left\{\tilde{B}, \tilde{W}^{0},\left(\tilde{H}_{1}^{0}+\tilde{H}_{2}^{0}\right) / \sqrt{2},\left(\tilde{H}_{1}^{0}-\tilde{H}_{2}^{0}\right) / \sqrt{2}\right\}$, with masses $\left\{M_{1}, M_{2},-\mu, \mu\right\}$. In order to decide which of the two higgsino combinations is the lightest, one has to include corrections of second order in $m_{Z}$. Then one obtains as mass eigenvalues

$$
\begin{gathered}
\left\{M_{1}-\frac{s_{W}^{2}\left(\mu \sin (2 \beta)+M_{1}\right) m_{Z}^{2}}{\mu^{2}-M_{1}^{2}}, M_{2}-\frac{c_{W}^{2}\left(\mu \sin (2 \beta)+M_{2}\right) m_{Z}^{2}}{\mu^{2}-M_{2}^{2}},\right. \\
\left.-\mu+\frac{(\sin (2 \beta)-1)\left(\mu+c_{W}^{2} M_{1}+s_{W}^{2} M_{2}\right) m_{Z}^{2}}{2\left(\mu+M_{1}\right)\left(\mu+M_{2}\right)}, \mu+\frac{(\sin (2 \beta)+1)\left(\mu-c_{W}^{2} M_{1}-s_{W}^{2} M_{2}\right) m_{Z}^{2}}{2\left(\mu-M_{1}\right)\left(\mu-M_{2}\right)}\right\},
\end{gathered}
$$

if the masses are not degenerate. Depending on the sign of $\mu$, the symmetric $(\mu<0)$ or the antisymmetric $(\mu>0)$ combination of the higgsino is the LSP for $|\mu| \ll M_{1}, M_{2}$. To be definite, we shall choose always $\mu>0$ in the following.

\section{Relevant processes and cross sections}

\subsection{Elastic scattering on fermions and the energy relaxation time}

Kinetic equilibrium of neutralinos in the late universe may be reached by scattering on light fermions like neutrinos and electrons. In the rest frame of the neutralino, the Mandelstam variables become $s=2 \omega m_{\chi}+m_{\chi}^{2}, \quad t=-2 \omega^{2}(1-\cos \vartheta)$ where $m_{\chi}$ is the mass of the lightest neutralino, $\omega$ is the initial energy of the lepton and $\vartheta$ is the scattering angle. We consider here only the case of a broken electroweak symmetry, i.e. the case of temperatures $T$ below the weak scale, when the following hierarchy holds

$$
\omega \ll m_{Z} \ll m_{\chi} .
$$

The assumption $m_{Z} \ll m_{\chi}$ leads also to several simplifications in the Higgs sector of the MSSM that we shall employ below. Additionally, we require that the neutralino mass parameters are not too degenerate, $\left|\mu-M_{1}\right|,\left|\mu-M_{2}\right|,\left|M_{2}-M_{1}\right| \gg m_{Z}$. We consider explicitly the case where the lightest neutralino is a bino or a higgsino and scatters on a neutrino. The case of a wino is almost identical to the one of the bino.

The bino as the LSP Using the approximations explained above, we obtain as the leading contribution to the total spin-averaged squared Feynman amplitude in the case of a bino [12]

$$
|\mathscr{M}|^{2}=\frac{e^{4} \omega^{2}(3-\cos (\vartheta)) M_{1}^{2}\left(\mu^{2}-2 c_{\beta}^{2} M_{1}^{2}+c_{2 \beta} M_{\tilde{v}}^{2}\right)^{2}}{2 c_{W}^{4}\left(\mu^{2}-M_{1}^{2}\right)^{2}\left(M_{1}^{2}-M_{\tilde{v}}^{2}\right)^{2}} .
$$

Here, we used neutrinos as scattering target and denoted by $M_{\tilde{v}}$ the sneutrino mass.

The energy relaxation time can be calculated as (see e.g. Ref. [13])

$$
\frac{1}{\tau_{\mathrm{rel}}}=\frac{N_{\mathrm{eff}}}{2 E_{k} m_{\chi}} \int_{0}^{\infty} d \omega \int d \Omega n_{0}(\omega)(\delta p)^{2}\left(\frac{d \sigma_{\mathrm{el}}}{d \Omega}\right)_{f_{L \chi}},
$$


where $E_{k}=(3 / 2) T$ is the mean kinetic energy of the neutralinos, $\delta p$ the neutralino momentum obtained in one scattering, $(\delta p)^{2}=2 \omega^{2}(1-\cos (\vartheta))$, and the number density of relativistic fermions with one polarization and energy $\omega$ is $n_{0}=\omega^{2} /\left(2 \pi^{2} e^{\omega / T}+1\right) \approx \omega^{2} e^{-\omega / T} /\left(2 \pi^{2}\right)$. Finally, the factor $N_{\text {eff }}$ counts the number of relevant relativistic degrees of freedom, weighted with the relative size of their cross-section compared to a neutrino. Combining the different contributions and performing the integrals gives

$$
\tau_{\text {rel }}=\frac{\pi^{3} c_{W}^{4} M_{1}\left(M_{\tilde{v}}^{2}-M_{1}^{2}\right)^{2}\left(\mu^{2}-M_{1}^{2}\right)^{2}}{25 N_{\mathrm{eff}} e^{4} T^{6}\left[c_{2 \beta} M_{\tilde{v}}^{2}+\mu^{2}-2 c_{\beta}^{2} M_{1}^{2}\right]^{2}} .
$$

The higgsino as the LSP Analogously to the case of the bino, the energy relaxation time for a Higgsino as lightest neutralinos follows as

$$
\tau_{\text {rel }}=\frac{\pi^{3} \mu^{3}\left(\mu-M_{1}\right)^{2}\left(\mu-M_{2}\right)^{2} s_{2 W}^{4}}{100 N_{\text {eff }} e^{4} T^{6} c_{2 \beta}^{2}\left(M_{1} c_{W}^{2}+M_{2} s_{W}^{2}-\mu\right)^{2}} .
$$

For the squared Feynman amplitude leading to the expression (2.5) for the energy relaxation time, confer [1].

\subsection{Elastic neutralino-neutralino scattering}

We use in this subsection again the assumptions (2.1), but denote now with $\omega$ the kinetic energy of the colliding neutralinos in their center of mass frame. Then the Mandelstam variables are $s=4\left(M_{\chi}^{2}+\omega^{2}\right)$ and $t=-2 \omega^{2}(1-\cos \vartheta)$.

The Bino as the LSP Neutralino-neutralino scattering can occur through $s$ and $t$ channel exchange of the $Z$ and the three neutral Higgs bosons.

Because of the hierarchy in the Higgs masses, $\mathscr{O}\left(M_{h}\right)=\mathscr{O}\left(m_{Z}\right) \ll \mathscr{O}\left(M_{H}\right)=\mathscr{O}\left(M_{A^{0}}\right)$, the $h$-exchange channel (2.6) is of order $\mathscr{O}\left(M_{S U S Y}^{0}\right)$, and dominates the self-scattering of superheavy neutralinos.

$$
\left|\mathscr{M}_{h \text {-exch }}\right|^{2}=\frac{8 e^{4} m_{Z}^{4} M_{1}^{4}\left(\mu \sin (2 \beta)+M_{1}\right)^{4} \tan ^{4}\left(\vartheta_{W}\right)}{M_{h}^{4}\left(\mu^{2}-M_{1}^{2}\right)^{4}},
$$

The other channels are of order $\mathscr{O}\left(m_{Z}^{4} / M_{S U S Y}^{4}\right)$ (or less), see [1] for exact expressions.

With $\left|\mathscr{M}_{\chi^{0} \chi^{0} \rightarrow \chi^{0} \chi^{0}}\right|^{2}=\left|\mathscr{M}_{h \text {-exch }}\right|^{2}$, the total cross section of neutralino-neutralino scattering follows as

$$
\sigma=\frac{e^{4} m_{Z}^{4} M_{1}^{2}\left(\mu \sin (2 \beta)+M_{1}\right)^{4} \tan ^{4}\left(\vartheta_{W}\right)}{16 M_{h}^{4} \pi\left(\mu^{2}-M_{1}^{2}\right)^{4}}
$$

The Higgsino as the LSP Analogously to the bino case, the leading contribution to higgsinohiggsino scattering is given by the exchange of the light, SM-like Higgs $h$. With

$$
\left|\mathscr{M}_{\chi^{0} \chi^{0} \rightarrow \chi^{0} \chi^{0}}\right|^{2}=\left|\mathscr{M}_{h \text {-exch }}\right|^{2}=\frac{e^{4}\left(c_{\beta}+s_{\beta}\right)^{8} \mu^{4} m_{Z}^{4}\left(M_{1} \cos ^{2}\left(\vartheta_{W}\right)-\mu+\sin ^{2}\left(\vartheta_{W}\right) M_{2}\right)^{4}}{2 c_{W}^{4} s_{W}^{4} M_{h}^{4}\left(\mu-M_{1}\right)^{4}\left(\mu-M_{2}\right)^{4}}
$$

the total cross section of neutralino-neutralino scattering follows as

$$
\sigma=\frac{e^{4}\left(c_{\beta}+s_{\beta}\right)^{8} \mu^{2} m_{Z}^{4}\left(M_{1} c_{W}^{2}+M_{2} s_{W}^{2}-\mu\right)^{4}}{256 \pi c_{W}^{4} s_{W}^{4} M_{h}^{4}\left(\mu-M_{1}\right)^{4}\left(\mu-M_{2}\right)^{4}} .
$$




\subsection{Annihilations}

The annihilations of neutralinos, $\chi^{0}+\chi^{0} \rightarrow X+Y$, have been studied in great detail. Annihilations of superheavy neutralinos are, in the bino case, dominated by the channels $Z+H, h+A, A+$ $H, W^{ \pm}+H^{\mp}$, since all other channels are suppressed by powers of $m_{Z} / M_{\text {SUSY }}$. In the higgsino case all bosonic channels contribute at leading order except annihilation into $Z^{0}+A^{0}$ and $h+H$. See [1] for the squared matrix elements of the dominating channels.

Annihilation into fermions are always suppressed.

\section{Summary}

We have suggested the lightest supersymmetric particle as a well-suited candidate for superheavy dark matter.

Our proposal can be falsified in the near future by the discovery of low-scale/split SUSY at the LHC. If this is not the case, then SHLSPs as DM particles may be the unique opportunity to connect SUSY to the physical world.

The prospects to detect DM in the form of stable superheavy neutralinos despite of their small number density and annihilation cross section will be discussed in a subsequent work [14].

\section{References}

[1] V. Berezinsky, M. Kachelriess and M. Aa. Solberg, Supersymmetric superheavy dark matter, arXiv:0810.3012 [hep-ph], submitted to Phys. Rev. D.

[2] G. Bertone, D. Hooper and J. Silk, Phys. Rept. 405, 279 (2005) [hep-ph/0404175].

[3] K. Griest and M. Kamionkowski, Phys. Rev. Lett. 64, 615 (1990); L. Hui, Phys. Rev. Lett. 86, 3467 (2001).

[4] J. Dunkley et al., arXiv:0803.0586 [astro-ph].

[5] V. Berezinsky, M. Kachelrieß and A. Vilenkin, Phys. Rev. Lett. 79, 4302 (1997) [astro-ph/9708217].

[6] V. A. Kuzmin and V. A. Rubakov, Phys. Atom. Nucl. 61, 1028 (1998) [Yad. Fiz. 61, 1122 (1998)].

[7] D. J. H. Chung, E. W. Kolb and A. Riotto, Phys. Rev. D 59, 023501 (1999); V. Kuzmin and I. Tkachev, JETP Lett. 68, 271 (1998); see also D. H. Lyth and D. Roberts, Phys. Rev. D 57, 7120 (1998).

[8] M. S. Chanowitz, M. A. Furman and I. Hinchliffe, Phys. Lett. B 78, 285 (1978); Nucl. Phys. B 153, 402 (1979).

[9] L. Girardello and M. T. Grisaru, Nucl. Phys. B 194, 65 (1982).

[10] A. Dobado, M. J. Herrero and S. Penaranda, arXiv:hep-ph/9806488, Eur. Phys. J. C 12, 673 (2000).

[11] J. F. Gunion and H. E. Haber, Phys. Rev. D 37, 2515 (1988). S. Mizuta and M. Yamaguchi, Phys. Lett. B 298, 120 (1993).

[12] For the calculation of amplitudes the package CompHEP was used, A. Pukhov et al., hep-ph/9908288.

[13] V. Berezinsky, V. Dokuchaev and Y. Eroshenko, Phys. Rev. D 68, 103003 (2003) [astro-ph/0301551].

[14] V. Berezinsky, V. Dokuchaev, Y. Eroshenko, M. Kachelrieß and M.Aa. Solberg, in preparation. 